, 


\title{
Ueber den
}

\section{Einfluss des Lichtes und der Wärme}

\author{
auf die
}

Retina des Frosches.

Von

Dr. Giuseppe Gradenigo jun.

Separat-Abdruck aus der, Allgem. Wr. mediz. Zeitung , 1885, Nr. 29 u. 30.

\section{' WIEN 1885.}

Im Selbstverlage des Verfassers. 

Digitized by the Internet Archive in 2014 
po (j.Gradenigo: Limflue nza della luce edel calore sulla relina della rana.

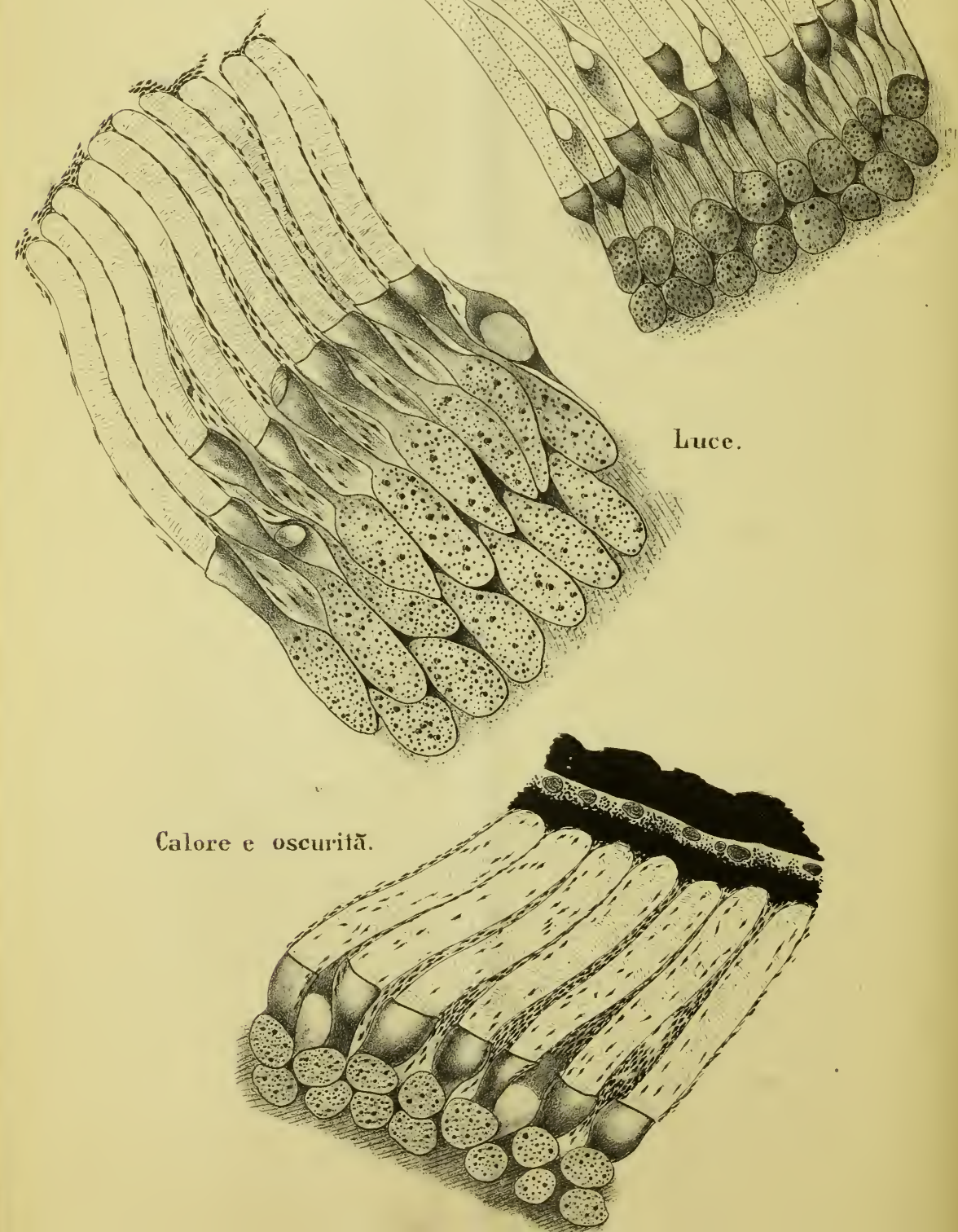


Seit beiläufig acht Jahren haben sich unsere Lehren über die Physiologie der Netzhaut ganz anders gestaltet. Eine Reihe von glänzenden Entdeckungen, an welche sich die Namen von Boll, Angelucci, Kühne und Eng elmann knüpfen, haben der Wissenschaft eine ganz neue Richtung, und den Forschungen über aie Physiologie des Sehactes einen Impuls gegeben, von welchen sich heute jedoch die Erfolge noch nicht vorhersehen lassen.

Zuerst lehrte Boll 1), dass bei Fröschen die Netzhaut eine besondere rothe Farbe besitzt, welche unter der Einwirkung des Lichtes verschwindet. Es wurde später constatirt, besonders in Folge der Arbeiten von $\mathrm{Kühn} \mathrm{e}^{2}$ ), dass dieser sogenannte ${ }_{n} \mathrm{Seh}-$ purpur", von wenigen Ausnahmen abgesehen, in allen stäbchenführenden Netzhäuten der Vertebraten vorkommt.

Schenk und Zuckerkandl ${ }^{3}$ ) wiesen die Sehfärbung in der menschlichen Retina nach, Fuchs und Velp on e $\mathbf{r}^{4}$ ) an neunund siebenmonatlichen menschlichen Föten, deren Augen niemals Licht empfangen.

Später beobachtete Boll, dass auch die Pigmentkörnchen der Epithel-Zellen, je nach der Intensität und Qualität des Lichtes, zwischen die Stäbchen und Zapfen mehr oder weniger eindringen. Angelucci5) bestätigte und verbreitete diese Lehre dahin, dass er eine Bewegung der Stäbchen-Aussenglieder, constatirte, indem sich diese Elemente unter dem Einflusse des weissen Lichtes ihrer Längsrichtung nach quetschen und sich in ihrem Querdurchmesser erheblich verbreiten.

In neuester Zeit hat van Genderen Stort, unter der Leitung von Th. W. Engel $\operatorname{mann}{ }^{6}$ ) nachgewiesen, dass die Zapfen-

1) Bo 11. "Berliner Monatsb.", 12. Nov., Accademia dei Lincei 3 Dic. 1876.

2) Kühne. In Hermann's Handb. d. Physiol., III. Bd., I. Abth.

3) "Allg. Wien. Med. Ztg. ${ }^{4}, 1877$, S. 221.

4) "Wiener Med. Wochenschr.", 1877.

5) Angel ucci. Ricerche istologiche sull' epitelio retinico dei Vertebr.

R. Accad. Lincei, $1878=$ Una nuova Teoria sulle Visione - Roma, 1884. 498, 1885. 
Innenglieder sich unter der Einwirkung des Lichtes verkürzen, und im Dunkeln verlängern; er hat ferner eine directe Abhängigkeit dieser Bewegungen vom Nervensystem beobachtet.

Soweit reichen die Erfolge der Versuche und Beobachtungen auf dem Gebiete des Einflusses des Lichtes über die empfindungsfähigen Elemente der Netzhaut. Es ist dabei besonders hervorzuheben, dass die Resultate dieser Beobachtungen, obwohl von den beiden letztgenannten Forschern über zwei verschiedene Elemente gepflogen, dennoch in der Modalität der Bewegungen der Stäbchen, respective der Zapfen, bezüglich der verschiedenen Arten von brechbaren Strahlen im Allgemeinen übereinstimmen

Es war in letzterer Zeit mein Bestreben, die Aufmerksamkeit auf die so wichtigen Befunde, wie sie von Angelucci und Engelman $n$ mitgetheilt wurden, zu richten, und ich sah mich veranlasst, die Untersuchungen über diesen Gegenstand zu wiederholen. Die Erfolge meiner Versuche bestätigten in erfreulicher Weise vollinhaltlich die Hauptsätze, welche von Angelucci und Engelmann aufgestellt worden sind; jedoch war ich in der Lage, manche neue Beobachtungen zu machen, welche, wie es mir scheint, von solchem Interesse sind, dass eine Mittheilung derselben nicht überflüssig sein dürfte.

Zuerst habe ich mit positiver Bestimmtheit ersehen, dass an der Froschretina unter der Einwirkung des Lichtes die StäbchenInnenglieder kürzer und dicker werden, in ähnlicher, wenn auch nicht so ausgedehnter und rascher Weise, wie Engelmann bei den Zapfen-Innengliedern die Beobachtung gemacht hat. Die sogenannten „äusseren Körner", welche, wie bekannt, mit den Stäbchen- und Zapfen-Innengliedern im Zusammenhange stehen, ändern ibre Gestalt, indem sie eine mehr ovale Form mit dem grössten Durchmesser in radiärer Richtung annehmen. Was mir ferner von Bedeutung erscheint, ist die erwiesene Thatsache, dass die Wärme in ganz ähnlicher Weise, wie das Licht, alle diese Bewegungen hervorzurufen im Stande ist.

Wenn man in einer Froschnetzhaut, deren Elemente mit $31 / 2 \%$ Salpetersäure fixirt und mit Haematoxylin gefärbt worden sind, die Stäbchen-Innenglieder betrachtet, bemerkt man, dass diese durch den Farbstoff an dem engeren Abschnitte dunkler gefärbt sind, während sie in dem breiteren, kelchartig erweiterten Stücke durch Haematoxylin wohl gefärbt sind, aber stets lichter erscheinen. Die Aussenglieder der Stäbchen bleiben gewöhnlich ungefärbt.

In einer Dunkelnetzhaut (d. i. eine Netzhaut von einem, durch mehrere Stunden im Dunkeln gehaltenen Frosche) bemerkt man, dass die Pigmentkörnchen nur die Kuppen der Aussenglieder der Stäbchen umgeben, die Stäbchen-Aussenglieder länger und schmäler sind als diejenigen einer dem Lichte ausgesetzten Netzhaut; die Zapfen 
sind mit verlängerten Innengliedern $z$ wischen den Stäbchen emporgestiegen. Die kelchartigen Ausbreitungen des Stäbchen-Innengliedes befinden sich nicht alle in derselben Ebene, d. h. die Linie, welche die Verbindungsstelle der Aussen- mit Innengliedern der Stäbchen bezeichnet, gestaltet sich in ihrem Verlaufe sehr unregelmässig, weil die Innenglieder, die einen mehr, die anderen weniger, hervorragen, gegen oben zu über eine wechselnde Strecke hinausreichend.

Eine Menge von Stäbchen-Innengliedern befindet sich ziemlich weit von der obbenannten Linie, ungefähr an der Hälfte der übrigen Stäbchen-Aussenglieder; von solchen wollen wir vorläufig absehen, und werden später speciell darauf zurückkommen.

Wenn man nur diejenigen kelchartigen Enden der StäbchenInnenglieder, welche in der obbenannten Linie sich befinden, oder nicht so weit entfernt von derselben bleiben, in Betracht zieht, so nimmt man wahr, dass alle dieselben anatomischen Charaktere besitzen, obwohl sie nicht in derselben Höhe sind. Die soeben beschriebenen Enden der Innenglieder sind durch engere fadenartige Abschnitte mit den respectiven Aussenkörnern verbunden, welche meistens eine kuglige Form darstellen.

In einer belichteten $\mathrm{Netzhaut}$ verändert sich bedeutend das Aussehen dieser Elemente. Während in der Dunkelnetzhaut im Allgemeinen die Entfernung zwischen den Verbindungsstellen der Aussen- und Innenglieder der Stäbchen und dem Kerne eine grössere ist, der Verbindungsfaden ein längerer und schmälerer, und das Korn mehr abgerundet erscheint, ist bei der belichteten Netzhaut die Entfernung eine geringere, der Verbindungsfaden ein kürzerer und dickerer und das Korn nimmt eine mehr ovale Form an.

Betrachtet man die Schichten im Ganzen, so kann man in den meisten Fällen bestimmte Veränderungen wahrnehmen, welche uns die Summe von den einzelnen Modificationen darstellen. Die Schichte der Stäbchen-Innenglieder und die Schichte der Körner verändern wechselseitig ihren Höhedurchmesser so, dass die Innengliederschichte in der Dunkelnetzhaut ebenso breit werden kann, wie die Aussenkörnerschichte, während die in der belichteten Netzhaut nur die Hälfte oder zwei Drittel der Höhe der Körnerschichte erreicht

Auch durch die ovale Form der Körner sieht deren Schichte bei schwacher Vergrösserung in der belichteten Netzhaut aus, als ob die durch die Aneinanderreihung der Stäbchen gebotene Streifung in die Aussenkörnerschichte sich hinein fortsetzen würde.

Man findet bei dem Frosche sowohl in der Dunkelnetzhaut, wie auch gewöhnlich in der belichteten Netzhaut Stäbchen-Innenglieder, welche beträchtlich in der Höhe zwischen den StäbchenAussengliedern liegen. Wie zahlreich diese Gebilde in der belichteten Netzhaut im Vergleiche zu jenen in der Dunkelnetzhaut 
erscheinenden, vorkommen, ist ungemein schwer zu erörtern, indem die Infiltration und die Anhäufung der Pigmentkörnchen zwischen den Stäbchen in den meisten Fällen so vollkommen die StäbchenAussenglieder verdeck $t$, dass das Vorbandensein dieser Innenglieder in der Höhe nicht zu erkennen ist. Jedoch, wenn das Thier nur kurze Zeit in der Dunkelheit geblieben ist, findet man in der Netzhaut, neben theilweiser zurückgebliebener Pigment-Infiltration, dass, während die Zapfen über die Schichte der Stäbchen-Innenglieder mit ihrem ganzen Höhedurchmesser hervorragen, die Stäbchen-Innengliederschichte und die Körnerschichte den Platz der belichteten Netzhaut nicht verlassen haben. In einer solchen Netzhaut kann nachgewiesen werden, dass die Stäbchen mit langen Innengliedern äusserst selten vorkommen. In jedem Falle, wenn auch selten, kommen die isolirten Innenglieder dieser besonderen Art von Stäbchen schmal und lang auch in der belichteten Netzhaut vor, und scheinen, ich möchte sagen, theilnahmslos an den von ähnlichen Elementen durchgeführten Bewegungen zu bleiben.

Ueber ihre functionelle Bedeutung im Allgemeinen kann ich bis jetzt nichts mit Bestimmtheit feststellen.

Um die constatirten Thatsachen in einem gemeinschaftlichen Begriff zu vereinbaren und erklären, muss man annehmen, dass die Veränderungen, welche an den äussersten Schichten der Netzhaut durch den Einfluss des Lichtes hervorgerufen werden, nicht in allen diesen Gebilden in gleichem Maasse und nicht in gleicher Zeit vorkommen. Beim Einfluss des Lichtes entwickelt sich am raschesten die Pigment-Infiltration; dann folgt die Zusammenziehung der Zapfen und der Stäbchen-Aussenglieder; zuletzt ist objectiv nachweisbar die Zusammenziehung der Stäbchen-Innenglieder und die Veränderung der Form der „äusseren Körner". Es gibt bestimmt noch andere Factoren, bis jetzt noch nicht genugsam eruirt, welche bezüglich des Verhaltens dieser Elemente in Betracht zu ziehen sind. Daher kommt es oft vor, dass man in einer und derselben Netzhaut verschiedene Lagen der empfindungsfähigen Elemente finden kann, was schon Engelman n bezüglich der Pigmentkörnerund Zapfenstellungen hervorgehoben hat.

Die von mir erwiesene Thatsache der von Stäbchen-Innenglieder ausgeführten Bewegungen unter der Einwirkung des Lichtes bildet einen nicht werthlosen Fortschritt in der Lehre der Physiologie der Netzhaut, so wie sie von Boll begründet wurde. Obwohl die Verlängerung und Verkürzung der Zapfen-Innenglieder, wie sie von Engelmann beschrieben wurde, am deutlichsten hervortritt, so sollen die Zapfen nur als einer der vielfältigen Factoren des Sehactes betrachtet werden.

Wenn man thatsächlich bemerkt, dass die Stäbchen in den Netzhäuten der best organisirten Thiere in überwiegender Zahl 
vorkommen, und wie sie sogar in bestimmten Netzhäuten die einzigen percipirenden Elemente darstellen, so darf die Wichtigkeit, welche ihnen im Sebacte zugeschrieben werden muss, nicht übersehen werden. Die Feststellung der functionellen Bewegungen der StäbchenInnenglieder, ganz ähnlich in ihrer Modalität jener von ZapfenInnenglieder, stimmt vollkommen überein mit den embryologischen und anatomischen Eigenschaften, die uns schon bekannt sind.

Dr. Oppenheimer ${ }^{1}$ ) hat in diesem Laboratorium nachgewiesen, dass die Stäbchen in ihrer Entwicklung vorwiegend die Zapfenform annehmen und dass sie aus diesen hervorgehen. Zuerst kommen im Embryo nur Zapfen vor, beim erwachsenen Thiere stellen die Zapfen mehr dem embryonalen Typus annähernde Gebilde dar.

Max Schultze ${ }^{2}$ ) hebt hervor, dass in den jungen Thieren der Durchmesser der Stäbchen an der Spitze so rasch abnimmt, dass der Unterschied zwischen Stäbchen und Zapfen ungemein schwer zu erkennen ist. Auch durch andere mikroskopische Details meint Max Schultze, dass es nur durch die Form und die Grösse einen essentiellen Unterschied zwischen Stäbchen und Zapfen gebe. In den Kaninchen und Meerschweinchen sehen die Innenglieder der Zapfen und der Stäbchen ganz ähnlich aus. Endlich ist es bekannt, dass in der Thierreihe beide Arten percipirender Elemente durch eine einzige derselben vertreten werden können.

Eine wichtige Frage stellt sich uns jetzt entgegen: nämlich wie sich diese Bewegung an Zapfen- und Stäbchen-Innengliedern am lebenden Protoplasma verhält. Engelmann bezeichnet schon die Bewegung des Zapfen-Innengliedes als eine solche, welche ähnlich der Bewegung des contractilen Protoplasma ist, und stützt sich hiebei auf Präparate, wo der jeweilige Zustand des Innengliedes durch $31 / 2 \%$ Salpetersäure fixirt wurde. An frischen Präparaten, welche ich durch Zerzupfen von Retinastücken in Kammerwasser erhalten habe, erhielt ich einige nähere Aufklärungen über die Art und Weise der Bewegung. Von einem Dunkelfrosch wurde die Netzhaut in seinem Kammerwasser untersucht, das Präparat durch Zerzupfen bei rubin-rothem Lichte angefertigt, und sogleich zur Untersuchung unter das Mikroskop gebracht. Es war an einem solchen Präparate der Sehpurpur und die einzelnen Elemente nach allgemein angenommenen Grundsätzen möglichst frisch und der Norm am nächsten stehend erhalten. Ereignete es sich, dass man ein passend isolirtes Stück eines Zapfens mit dem OpticusEllipsoide und Innengliede erhielt, so zeigte sich wiederholt Folgendes: Das Innenglied, welches an dem Opticus-Ellipsoid hing, war,

1) Oppenheimer. Mittheilungen aus dem embryol. Institute von Dr. Schenk, I. Bd., II. Heft.

2) Max Schultze in Stricker's Handbuch der Lehre von den Gereben 1872. V. Lieferung, Capitel XXXVI. 
wie schon Schwalbe ${ }^{1}$ ) hervorhebt, bald homogen, bald schwach granulirt. Veränderungen an demselben im frischen Zustande waren. nicht leicht an allen oder an den meisten Stücken von Innengliedern zu beobachten; es gelang aber trotzdem, einige Bewegungserscheinungen an einzelnen isolirten Zapfen mit ihren Innengliedern wahrzunehmen, welche darin bestanden, dass dieselben abwechselnd d ünner und dicker wurden, bis endlich das unbewegliche Innenglied eine gewisse Länge erreicht hat, welche es bleibend beibehielt.

Wenn wir aus dieser Beobachtung einen Rückschluss auf die Bewegungsform uns erlauben, so geht daraus hervor, dass die Bewegung, welche eine Verkürzung nach sich zieht, nicht mit einem Male auftritt, sondern die Verkürzung des Innengliedes scheint in der Weise zu Stande zu kommen, dass abwechselnd vor dem Erreichen einer gewissen Kürze das Innenglied bald länger, bald kürzer, beziehungsweise dünner oder dicker wird.

Auf den Zusammenhang, welcher zwischen Innengliedern und Aussenkörnern besteht, ebenso wie auf die isolirten äusseren Körner, suchte ich an meinen frischen Zupfpräparaten die Aufmerksamkeit hinzuwenden. Ich war öfter in die Lage versetzt, isolirte ZapfenInnenglieder mit den Körnern in Zusammenhang $\mathrm{zu}$ sehen und fand dabei, dass bei der stattgehabten Veränderung des Innengliedes, auch das bezügliche Korn manche Bewegungen durchmachte, welche sich derart manifestirten, dass am Rande desselben kleine tangentiale Abschnitte sich körnchenfrei zeigten, ja zuweilen über das Niveau erhoben waren. Der grosse Kern in derselben blieb, so weit meine Beobachtungen reichen, unverändert. Wenn also eine Verkürzung oder Verlängerung der Innenglieder und eine Bewegung in den Körnern zugleich stattfindet, so ist es leicht ersichtlich, dass mit der Bewegung der Innenglieder auch eine solche in dem Protoplasma der Körner vor sich gehen wird. Somit kann man annehmen, dass die Körnchen in den Körnern, aus diesen in die Innenglieder sich fortsetzen; wo aber die Protoplasma-Verschiebung fehlt, da wird das Innenglied ein homogenes Aussehen erlangen. Es ist daher die von den Histologen angegebene Beschreibung, dass die Innenglieder der Zapfen und Stäbchen bald körnig, bald homogen erscheinen, aus dem Angeführten erklärlich.

Diese Thatsachen lassen mich vermuthen, dass die Bewegung in radiärer Richtung unter der Einwirkung des Lichtes in grösserer Ausdehnung als den Stäbchen-Aussengliedern und den Zapfen- und Stäbchen-Innengliedern entspricht, statthat; und bei besonderen Hilfsmitteln dürfte die Beobachtung ergeben, dass die Bewegungs-Er-

1) Schwalbe in Graefe's und Saemisch' Handbuch d. Augenheilkunde. I. Bd., IV. Capitel. 
scheinungen in radiärer Richtung, innerhalb der Retina, vielleicht auch auf die weiteren Lagen derselben sich erstrecken.

Diese merkw ürdigen Thatsachen an den Gebilden der Retina unter verschiedenen Lichteinflüssen, gehen, wie so eben erörtert, mit Formveränderungen der Zellen in dem Stratum Pigmento $s$ u m einher. In auffälliger Weise ergab sich im Verlaufe meiner Untersuchungen, dass diese Bewegungserscheinungen, sowohl die an den Pigmentzellen, als auch diejenigen an den Innengliedern der Zapfen und der Stäbchen, nicht nur unter dem Einflusse des Lichtes zu beobachten sind, sondern bei der Dunkel-Retina kann eine Erwärmung denselben Effect zeigen, wie man ihn an der belichteten Netzhaut beobachtet.

Wenn ich einen Frosch längere Zeit im Dunkeln hielt, die Augen desselben rasch enucleirte (bei rother Beleuchtung), und eines derselben einer Temperatur von $30-36^{\circ} \mathrm{C}$. aussetzte, so waren die Innenglieder kürzer als in dem anderen Auge, welches im Dunkeln war, ohne erwärmt worden zu sein. Ébenso verhielten sich die Pigmentzellen bei dem ersteren, in ähnlicher Weise wie es dem Verhalten derselben bei der belichteten Retina entspricht, obgleich. das Auge im Dunkeln blieb, bis die $31 / 2 \%$ Salpetersäure-Lösung genügend bis zur Fixirung der Elemente auf das Auge eingewirkt hatte.

Ich stellte den Versuch wiederholt auch in der W eise an, dass ich einen Frosch in einer Trockenkammer hielt, welche dunkel gemacht wurde, und selbe überdies in ein Dunkelzimmer gebracht wurde, bei einer Temperatur $\left(30^{\circ} \mathrm{C}\right.$.), welche nach längerem Einflusse an dem Thiere Wärmestarre hervorzubringen im Stande war. Das Auge wurde zur Untersuchung genommen, in dem Momente, als man bereits Wärmestarre an den Muskeln jener Seite der Extremitäten, welche mit dem Boden des Gefässes in Berührung stand, nachweisen konnte. Zuweilen wurde das Auge früher zur Untersuchung verwendet. Auf die Vertheilung des Fuscins in den Epithelzellen und in ihren Fortsätzen wurde bereits von $K \ddot{~ u ̈ h n e ~ d i e ~ W a ̈ r m e ~}$ als geltend gemacht, und war er im Stande nachzuweisen, besonders an curarisirten Fröschen, dass bei einer Temperatur-Erhöhung von etwa $30^{\circ}$ C. ein Vorschreiten des Pigmentes zu den Innengliedern bei Dunkel-Fröschen möglich ist. Meine Untersuchungen belehren mich, dass die Fuscinkörnchen bei höherer Temperatur an DunkelFröschen constant bis vor die Membrana Limitans externa reichen, und dass sie nicht immer in einer Linie, aus Fuscinnadeln gebildet, angeordnet sind, sondern die Art und Weise der Anhäufung dieser Pigmentkörnchen steht mit ihren krystallinischen Formen in verschiedenem Zusammenhange. Zuweilen ist das Pigment so dicht angeordnet, dass man mit Bestimmtheit - ohne es früher anderweitig erfahren zu haben - aus der Pigmentvertheilung nicht ur- 
theilen kann, ob man es nicht mit Pigmentzellen einer belichteten Retina zu thun hat. Von ähnlicher Wirkung war die erhöhte Tem. peratur auch für die Innenglieder der Zapfen und Stäbchen, so dass dieselben ähnlich verkürzt vorkommen, wie es bei der belichteten Retina der Fall ist.

Zum Schlusse ist für uns von Interesse, wenn wir einen raschen Blick in die ophthalmologische Literatur werfen, und dabei consta. tiren, wie mehrere Gebilde, deren functionelle Bedeutung wir oben erörtert haben, schon früher von anderen Autoren als anatomische Thatsachen anerkannt und beschrieben worden sind.

Schwalbe ${ }^{1}$ ) war es zuerst, der an der Froschretina die Stäbchen mit den langen Innengliedern als eine merkwürdige zweite Art von Stäbchen beschrieben und abgebildet hat; - was später von K ühne, welcher jenen Stäbchen eine bestimmte grüne Farbe zuschreibt, und von R anvier bestätigt wurde. - Von diesen Gebilden kann, nach dem heutigen Stande unseres Wissens, nicht mit Bestimmtheit behauptet werden, ob ihnen ein functioneller, oder rein anatomischer Werth zukommt, oder ob man sie mit embryonalen und regenerativen Vorgängen in Zusammenhang bringen soll. Von den gleich zu besprechenden Gebilden jedoch, die bisher als einfache anatomische Befunde beschrieben worden sind, ist mit Sicherheit anzunehmen, dass sie zu der Einwirkung des Lichtes in inniger Beziehung stehen.

Landolt ${ }^{2}$ ) bemerkt, wie die Zapfen an der Froschretina selten in derselben Ebene sich befinden; und in Fig. $3 b$ seiner $\mathrm{Ab}$ bildungen stellt er uns zwei Zapfen nebeneinander dar, der eine mit längerem, der andere mit kürzerem Innengliede. $L$ and olt bezeichnet diese Zapfen als Doppelzapfen; doch ist ihre Verbindung auf dem betreffenden Bilde nicht angegeben, und man kann sie ganz gut für Zapfen mit verlängertem und zusammengezogenen Innengliede ansehen.

Betreffs der „äusseren Körner" hebe ich nur zwei merkwürdige Beobachtungen hervor. Landolt, welcher sich wahrschein. lich in seinen Untersuchungen nur mit belichteten Froschnetzhäuten befasste, bemerkt nämlich wie die äusseren Körner an dem peripherischen Theil der Netzhaut nicht mehr so spindelförmig lang gezogen, wie im Centrum, sondern kurz, an beiden Polen abgerundet sind. Diese Erscheinungen an den äusseren Körnern sind mit dem Einflusse des Lichtes in Verbindung zu bringen. Thatsächlich sind die Charaktere der belichteten Retina nur an centralen, nicht aber heilkunde.

1) Schwalbe - in Graefe's und Saemisch' Handbuch f. Augen.

2) Landolt. Beitrag zur Anatomie der Retina von Froseh, Salamander und Triton. Archiv für mikroskop. Anatomie. Bd. 7, 1871, S. 84 . 
an den mehr peripherischen Theilen $\mathrm{zu}$ suchen, wie dies auch Angel ucci bemerkt.

Czerny, ${ }^{1}$ ) welcher mehrere Versuche zur Blendung durch Sonnenlicht auch an der Froschretina im Laboratorium Brü cke's angestellt hat, bemerkt, wie die Elemente der A ussenkörnerschichte an den geblendeten Stellen, d. i. wo direct das Licht auf die Netzhaut eingewirkt hat, mehr oblong bis spindelförmig, während sie an den übrigen Partien mehr kuglig sind. Diese ovalen Formen der Körner bedeuten für C z e rny einen pathologischen Vorgang, für mich sind sie eine functionelle Thatsache.

Die hier mitgetheilten Untersuchungen habe ich im Schenk'schen Laboratorium in Wien ausgeführt. Da Prof. S chenk mir bei meinen Arbeiten mit gutem Rathe in der liebenswürdigsten Weise immer beigestanden ist, spreche ich ihm hier meinen besten Dank aus.

1) Czerny. LVI. Bd. d. Sitzungsb. d. k. Akad. d. Wissensch. Jahrg. 1867. 

- 
